\title{
Detection of Microaneurysms in Color Retinal Images Using Multi-orientation Sum of Matched Filter
}

\author{
Qin Li, Ruixiang Lu, Shaoguang Miao, Jane You
}

\begin{abstract}
This paper presents a new method to detect retinal microaneurysms, which are the first sign of diabetic retinopathy (DR). The methods of automatic microaneurysm detection can be generally classified into two groups: filtering based methods and supervised methods. The detection accuracy of the filtering based methods is affected by the background noises and the small vessels. The supervised methods generally have better accuracy by feature sets extraction and training. But the computational cost of feature sets extraction is very high, which makes the supervised methods not suitable for large population screening. And the accuracy of supervised methods will affected by training samples. This paper presents a new method to detect retinal microaneurysms using Multi-orientation Sum of Matched Filter (MSMF), which can well suppress the affection of background noises and the small vessels. Experimental results show that our method is fast and effective in microaneurysm detection.
\end{abstract}

Keywords: Microaneurysms detection $\cdot$ Color retinal image $\cdot$ Matched filter

\author{
Qin $\operatorname{Li}(\square)$ \\ Lab of Medical Devices, Shenzhen Academy of Metrology \& Quality Inspection, \\ Shenzhen, China \\ e-mail: kenneth_lee_qin@qq.com \\ Ruixiang $\mathrm{Lu}$ \\ Lab of Medical Devices, Shenzhen Academy of Metrology \& Quality Inspection, \\ Shenzhen, China \\ Shaoguang Miao \\ Department of Computing, the Hong Kong Polytechnic University, Hong Kong \\ Jane You \\ Department of Computing, the Hong Kong Polytechnic University, Hong Kong
}




\section{Introduction}

Diabetic retinopathy (DR) is a common and severe complication of long-term diabetes which damages the retina and cause blindness [1]. Color fundus camera is an easily operated tool to capture human retina so that the color retinal images are suitable for large population screen. The computer aided diagnosis (CAD) on retinal images can help eye specialists to screen larger populations. Since microaneurysms are regarded as the first signs of DR [2], the automatic detection of microaneurysms is very important. Generally, the methods of automatic microaneurysms detection can be classified into two groups: filtering based methods and supervised methods.

Microaneurysms can be regarded as local minimum in the greyscale image. Therefore they can be detected by different filters. In [5], Walter et al. proposed morphological diameter opening and closing filters to detect microaneurysms by defining the diameter of a connected region as its max extension. In [6], Sinthanayothin et al. proposed a modified morphological top-hat filter and segmented microaneurysms by region growing. In [3][4], Spencer et al. and Frame et al. first suppressed the vessels by morphological multi-orientation closing, then the microaneurysms are detected by applying a 2D Gaussian matched filter on the Fluorescein Angiography images. In [10], Niemeijer et al. extended this SpencerFrame scheme on color retinal images by a shade correction operation to first erase the bright lesions. In [7], Fleming et al. improved the 2D Gaussian matched filter by local contrast normalization. In [11], Fujita et al. proposed a modified double ring filter for microaneurysm detection.

In color retinal images, the microaneurysms are not as clear as in Fluorescein Angiography images. The detection accuracy of the filtering based methods is affected by the background noises. And, because microaneurysms are often surrounded by small vessels, they could be "merged" into small vessels during filtering operation. And, in color retinal images, the greyscale distribution along vessel orientation are often discontinuous so that the small vessels are easily misclassified as microaneurysms even the vessels are suppressed by morphological operation [3][4][10].

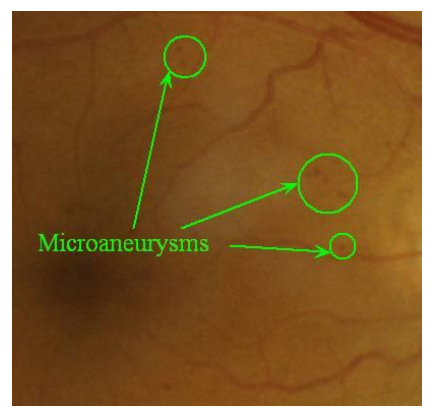

Fig. 1 Examples of microaneurysms 
The supervised methods generally have better accuracy by feature sets extraction and training. Other than local greyscale distribution, more features such as geometrical feature, color feature and statistical feature are extracted to improve the microaneurysm detection accuracy. In [8], Gardner et al. proposed an artificial neural network based scheme for microaneurysm detection. In [9], Zhang et al. extracted statistical feature using PCA and then detected microaneurysms using SVM classifier. In [12], Zhang et al. defined a feature sets using 31 different features and then microaneurysms are classified by a discrimination table.

However, the computational cost of feature sets extraction is very high, which makes the supervised methods not suitable for large population screening. And, the accuracy of supervised methods is highly related to the selection of training samples. Furthermore, the manually labelling of training sample is a very challenging work because microaneurysms have blurry edges and huge amount of microaneurysm samples have to be labelled to make the classifiers work well.

This paper presents a new method to detect retinal microaneurysms using Multi-orientation Sum of Matched Filter (MSMF), which can well suppress the affection of background noises and the small vessels. The rest of this paper is organized as following. Section 2 describes MSMF in details. Experimental results and conclusions are given in section $3 \& 4$ respectively.

\section{Method}

This section describes our method to detect retinal microaneurysms. First, the same with Niemeijer et al. [10], bright lesions and vessels are suppressed by shade correction and morphological operation. Then, the microaneurysms are enhanced by MSMF.

In [10], Niemeijer et al. defined a 2D Gaussian matched filter as described in Equation (1) as

$$
G(x, y)=\frac{1}{2 \pi \sigma^{2}} \exp \left(\frac{-x^{2}+y^{2}}{2 \sigma^{2}}\right)
$$

where $\sigma$ is the standard deviation of the Gaussian function. This is an isotropic filter. This filter is good at enhancing microaneurysms, but it enhances vessels as well. Even most of the vessels are well suppressed by the morphological operation, there are some small vessels can not be well suppressed. These small vessels generally have discontinuous greyscale distribution along vessel orientation and are easily mis-classified as microaneurysms. Furthermore, false negative detection could be caused by this filter because microaneurysms could be "merged" into surrounding small vessels during filtering operation.

In order to produce better microaneurysm enhancement, we designed the Multiorientation Sum of Matched Filter (MSMF) where the matched filter is defined on the anisotropic Gaussian function defined in Equation (2) as 


$$
m(x, y)=\frac{x^{2}-\sigma^{2}}{\sqrt{2 \pi} \sigma^{5}} \exp \left(\frac{-x^{2}}{2 \sigma^{2}}\right), \quad \text { for }|x| \leq 3 \sigma, \quad|y| \leq L / 2
$$

where $\sigma$ is the standard deviation of the Gaussian function, $L$ is the length of the neighborhood along $y$-axis to smooth noise. This smoothing can help to enhance the continuity of vessels so that small vessels are not mis-classified as microaneurysms. And, in order to produce strong response at the centre of a microaneurysm, we will rotate $m(x, y)$ to different orientations as defined by Equation (3) where $\phi$ represents orientation. Then the MSMF is defined in Equation (4) where $f(x, y)$ represents the green channel of the original retinal image and * represents convolution. Fig.2(a) shows the matched filter in 3D view and Fig.2(b) shows the matched filter with different $\sigma$ and orientations.

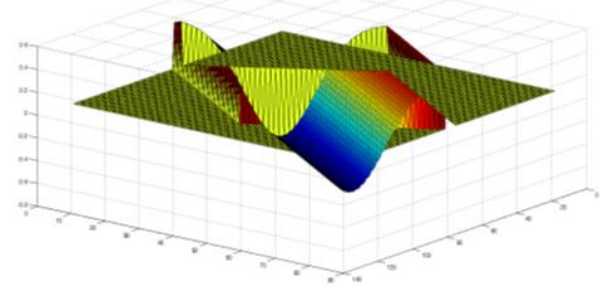

(a)

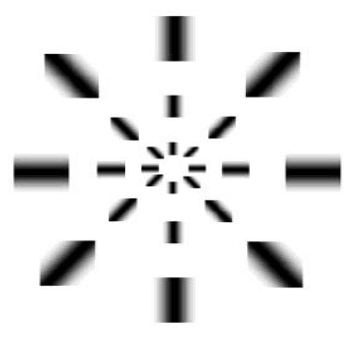

(b)

Fig. 2 Proposed matched filter.

$$
\begin{gathered}
\left\{\begin{array}{l}
m^{\phi}\left(x^{\prime}, y^{\prime}\right)=m(x, y) \\
x^{\prime}=x \cos \phi+y \sin \phi \\
y^{\prime}=y \cos \phi-x \sin \phi
\end{array}\right. \\
R(x, y)=\sum_{\phi} f(x, y) * m^{\phi}(x, y), \quad \phi=0, \pi / 12, \pi / 6, \ldots, 11 \pi / 12
\end{gathered}
$$

Without lost of the generality, we analyze the matched filter in 1-D case. Both the cross-sections of a microaneurysm and of a vessel can be defined by Equation (5) where $w$ is the width of the cross-section. Then the convolution result of the matched filter can be derived by Equation (6). $g_{\sigma}^{\prime}$ and $g_{\sigma}^{\prime \prime}$ represent the first and second derivative of Gaussian function respectively.

$$
v(x)=\left\{\begin{array}{cc}
-h, & |x| \leq w \\
0, & |x|>w
\end{array}\right.
$$




$$
\begin{aligned}
R(x) & =m(x) * v(x) \\
& =\int_{-\infty}^{\infty} g_{\sigma}^{\prime \prime}(t) v(x-t) d t \\
& =\int_{-\infty}^{x-w} g_{\sigma}^{\prime \prime}(t) \cdot 0 d t+\int_{x-w}^{x+w} g_{\sigma}^{\prime \prime}(t) \cdot(-h) d t+\int_{x+w}^{\infty} g_{\sigma}^{\prime \prime}(t) \cdot 0 d t \\
& =h\left(g_{\sigma}^{\prime}(x-w)-g_{\sigma}^{\prime}(x+w)\right)
\end{aligned}
$$

Then the centre response can be derived by Equation (7).

$$
\begin{aligned}
R(0,0) & =\sum_{\phi} 2 \cdot h\left(g_{\sigma}^{\prime}(w)\right) \\
& =24 \cdot h\left(g_{\sigma}^{\prime}(w)\right)
\end{aligned}
$$

Because the shape of a microaneurysm is a circle, its cross-sections are the same at all orientations. But cross-sections of the vessels are different at different orientations. The convolution results are close to zero at the orientations near the vessel orientation. Therefore the centre response of a microaneurysm is enhanced and the vessel response is suppressed.

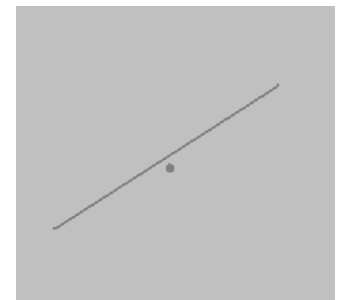

(a)

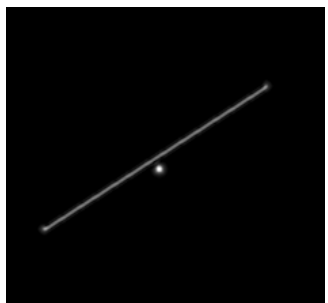

(b)

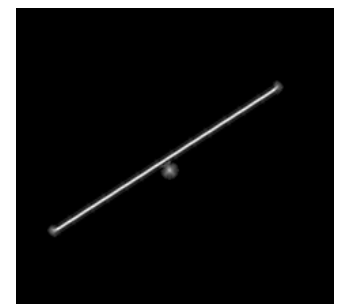

(c)

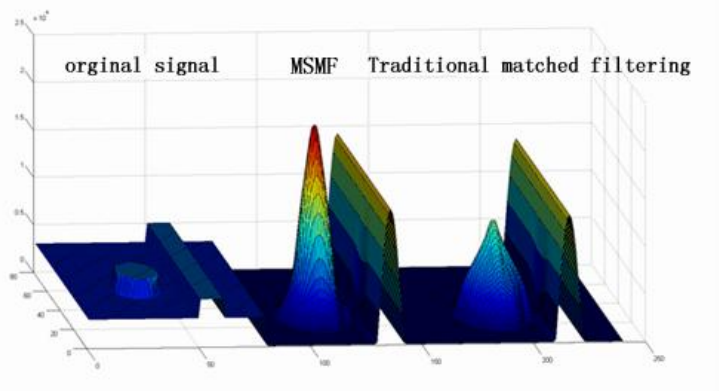

(d)

Fig. 3 MSMF demonstration. (a) the original signal; (b) the convolution result of MSMF; (c) the convolution result of the traditional matched filtering; (d) (a) (c) in 3D view. 


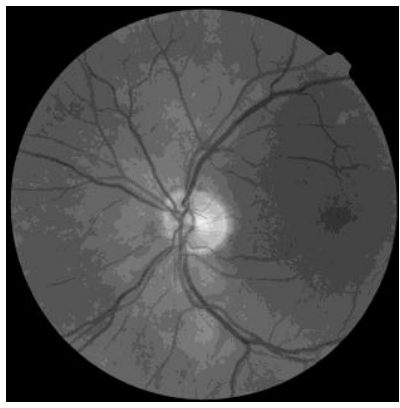

(a)

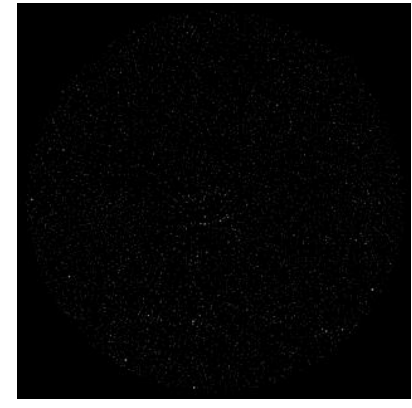

(b)

Fig. 4 (a) A retinal image from ROC database [11]; (b) the convolution result of MSMF.
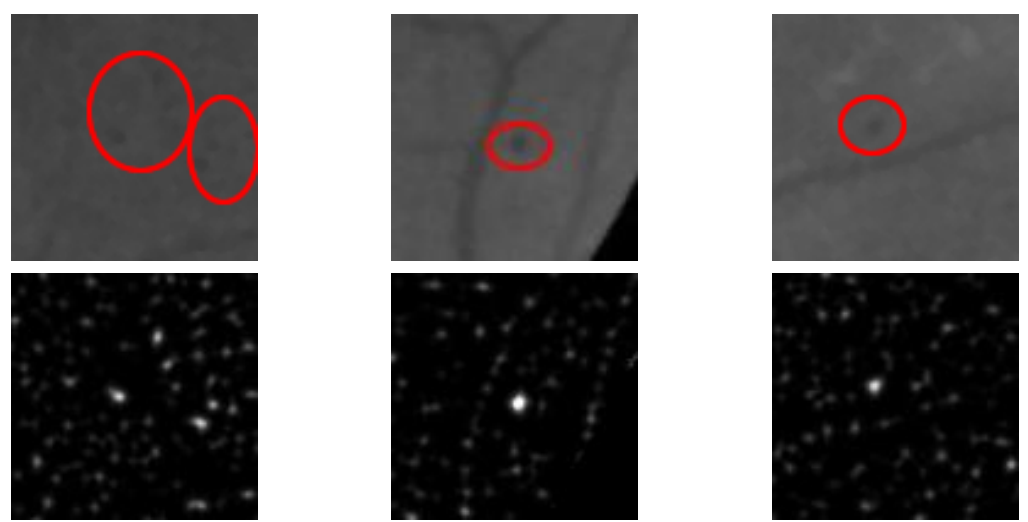

Fig. 5 MSMF examples: the dark dots insides red circles are microaneurysms

Fig. 3 demonstrates the performance of the MSMF using a simulated retinal image. Fig. 3(a) shows the original signal; Fig. 3(b) shows the convolution result of MSMF; Fig. 3(c) shows the convolution result of the traditional matched filtering [10]; Fig. 3(d) shows (a) (c) in 3D view. The centre response of a microaneurysm is well enhanced as shown in Fig. 3(b) \& (d). And, from Fig. 3(c), we can see that the false negative detection could be caused by this filter because microaneurysms could be "merged" into surrounding small vessels during filtering operation. This false negative detection can be avoided by MSMF as shown in Fig. 3(b). Fig. 4 shows the performance of MSMF using an image from ROC database [11]. To allow a better view, we cropped and zoomed-in part of the images, as in Fig. 5. From Fig. $4 \& 5$, we can see that the microaneurysms are well enhanced while the vessels are suppressed. 


\section{Experimental results}

The proposed method is tested on a publicly available database ROC [11]. ROC database provides 50 retinal images with manually labeled ground truth. We compare MSMF with the traditional matched filter [10] and a supervised method [12].

Fig. 6 is the FROC produced by plotting lesion sensitivity against average false positives per image. Table 1 lists the sensitivity of $1,2,3,4,5,7$ and 8 false positives per image. Table 2 records the execution time of different methods on an Intel core i5 computer.

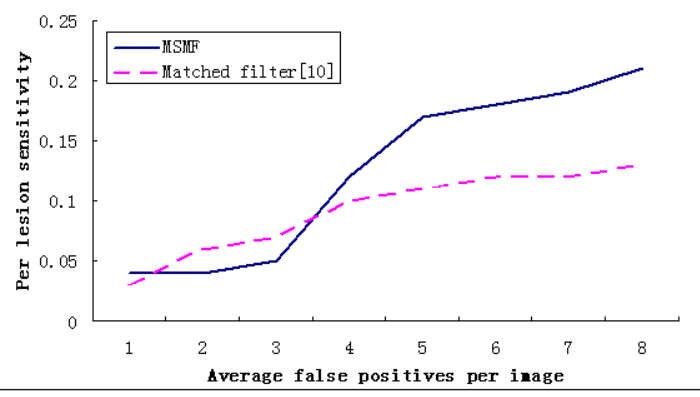

Fig. 6 Plot of FROC.

Table 1 Per lesion sensitivity of different false positives per image

\begin{tabular}{|l|l|l|l|l|l|l|l|l|}
\hline FPs/Scan & 1 & 2 & 3 & 4 & 5 & 6 & 7 & 8 \\
\hline MSMF & 0.04 & 0.04 & 0.05 & 0.12 & 0.17 & 0.18 & 0.19 & 0.21 \\
\hline Matched filter [10] & 0.03 & 0.06 & 0.07 & 0.10 & 0.11 & 0.12 & 0.12 & 0.13 \\
\hline Supervised [12] & 0.19 & 0.27 & 0.28 & 0.31 & 0.31 & 0.32 & 0.33 & 0.35 \\
\hline
\end{tabular}

Table 2 Execution time

\begin{tabular}{|l|l|}
\hline MSMF & 9 seconds \\
\hline Matched filter [10] & 4 seconds \\
\hline Supervised [12] & 273 seconds \\
\hline
\end{tabular}

\section{Conclusions and future works}

This paper presents a new method to detect retinal microaneurysms using MSMF, which can well suppress the affection of background noises and the small vessels. Experimental results show that our method is fast and effective in microaneurysm detection. 
However, the accuracy of MSMF is not as good as the supervised method [12]. In the future, we will do more research on fast feature extraction to save the time consuming of the supervised method. And, we will do more research on semisupervised learning to make the training sample labeling more efficient.

Acknowledgements. This work is supported by the Guangdong Natural Science Foundation (No. S2012040007988).

\section{References}

1. Bresnick GH, Mukamel DB, Dickinson JC, and Cole DR (2000) A screening approach to the surveillance of patients with diabetes for the presence of vision-threatening retinopathy. Opthalmology 107:19-24.

2. Philip S, Fleming AD, Goatman KA, Fonseca S, McNamee P, et al. (2007) The efficacy of automated "disease/no disease" grading for diabetic retinopathy in a systematic screening programme. Br. J. Ophthalmol. 91:1512-1517.

3. Spencer T, Olson J, McHardy K, Sharp P, and Forrester J (1996) An image processing strategy for the segmentation and quantification in fluorescein angiograms of the ocular fundus. Comput. Biomed. Res. 29: 284-302.

4. Frame A, Undrill P, Cree M, Olson J, McHardy K at al. (1998) A comparison of computer based classification methods applied to the detection of microaneurysms in ophthalmic fluorescein angiograms. Comput. Biol. Med. 28: 225-238.

5. Walter T, Klein JC, Massin P, and Erginay A (2002) A contribution of image processing to the diagnosis of diabetic retinopathy detection of exudates in color fundus images of the human retina. IEEE Transactions on Medical Imaging 21: 2002.

6. Sinthanayothin C, Boyce J, Williamson T, Cook H, Mensah E, et al. (2002) Automated detection of diabetic retinopathy on digital fundus images. Diabetic Med. 19: 105-112.

7. Fleming AD, Philip S, Goatman KA, Olson JA, and Sharp PF, Automated Microaneurysm Detection Using Local Contrast Normalization and Local Vessel Detection. IEEE Transactions on Medical Imaging 5: 1223-1232.

8. Gardner GG, Keating D, Williamson TH, and Elliott AT, (1996) Automatic detection of diabetic retinopathy using an artificial neural network: a screening tool, Brit. J. Ophthalmol. 80: 940-944.

9. Zhang X, Chutatape O (2005) Top-Down and Bottom-Up Strategies in Lesion Detection of Background Diabetic Retinopathy. CVPR 2; 422-428.

10. Niemeijer M, Ginneken B, Staal J,. Suttorp-Schulten MSA, Abramoff MD (2005) Automatic detection of red lesions in digital color fundus photographs. IEEE Trans. Medical Imaging 24: 584-592.

11. Niemeijer M, Ginneken B, Cree MJ, You J, Fujita H et al. (2010) Retinopathy Online Challenge: Automatic Detection of Microaneurysms in Digital Color Fundus Photographs. IEEE Transactions on Medical Imaging 29: 185-195.

12. Zhang B, Wu XQ, You J, Li Q, Karray F (2010) Detection of microaneurysms using multiscale correlation coefficients. Pattern Recognition 43: 2237-2248. 